\title{
Modelling of Measurement Error for Vibrational Displacement Based on the Blurring Analysis of a Round Mark Image
}

\author{
Alexey Grigoriev ${ }^{1 *}$, Evgeniya Danilova ${ }^{2}, V_{\text {Vailiy }} \operatorname{Trusov}^{3}$, Michail Miheev $^{4}$, Marina Uhanova $^{5}$ \\ ${ }^{1-3}$ Penza State University, Penza, Russia \\ ${ }^{4}$ Penza State Technological University, Penza, Russia \\ ${ }^{5}$ Riga Technical University, Riga, Latvia
}

\begin{abstract}
With the aim to compare methods for counting the number of lines of a raster matrix, intersecting a round mark image, and a number of pixels belonging to this image for measuring its radius, a numerical simulation is carried out in the present article. It is proved that the application of the method for counting the number of pixels belonging to the image of the round mark allows obtaining more than 30 times gain in the accuracy of this image radius measurement using the same equipment. The formulas proposed in the article are used for software implementation of non-contact vibration measurement systems.
\end{abstract}

Keywords - Error, pixel, raster, round mark, sampling.

\section{INTRODUCTION}

Measurement of a vector field of vibrational displacements of material points on the control object surface is an important means for obtaining initial data for vibration diagnostics of technical objects. Methods for such monitoring are divided into contact and non-contact ones. Contact methods, including such a promising one as fiber-optic [1], [2], do not provide vibration measurement, which frequency exceeds seven to eight kilohertz. Non-contact methods of laser Doppler and interferometric vibrometry require expensive bulky equipment, and do not provide vibration direction measurement. At present, a method for measuring a vector field of vibrational displacements on the surface of a control object by applying a round mark matrix to this surface, and then by automatically analysing the parameters of the vibrational blurring of the elements of this matrix, is being developed.

An image of the round mark is halftone. To process this image, it is converted to a binary one. Depending on the purpose of the measurement results, this transformation can be performed by the traditional threshold level method, or by applying a negative-contour classification of pixels in the halftone image [2]-[5]. Simulation of the blurry image track of the round mark with its coplanar and orthogonal vibrational displacements is explained in [3]. The structure for measuring technique of vibration parameters along the track of the image blurring of the round mark is proposed in [4], [6], [7].

A further development of the technique for analysing the vibration blurring of a round mark image, in an arbitrary direction and at an arbitrary angle to the object plane, is carried out in [5]-[9]. The advantage of this technique is that it allows measuring not only vibration magnitude, but also its direction. The drawbacks of this method are the limitation in increasing the measurement accuracy as the error of a round mark image sampling cannot be less than half of the raster unit.

A method for measuring the radius of a round mark image, based on counting a number of pixels, belonging to this image, is described in [10]. However, the requirements for the measurement accuracy are not justified there; the error of image sampling is not considered in this work, but is considered to be a general error of the system. A method for measuring the vibrational displacement of the investigated material point along the $z$-coordinate, based on measuring a radius increment of the round mark image, is substantiated in [11]-[14]. This paper also proves the linearity of the dependence of the measuring signal (the vibrational radius increment of the round mark image) on the measured value (amplitude of the vibrational displacement of the material point on the surface of the control object along the $z$-coordinate). However, this paper does not provide the analysis of the radius measurement error of the round mark image.

The present article provides the estimation of the sampling error when measuring radius of the round mark image by the traditional way of counting the number of lines of a raster matrix that intersect this image, and when measuring the radius of the round mark image by the method of counting the number of pixels of the raster matrix belonging to this image. A comparative assessment of the methods is carried out by the method of mathematical modelling. Numerical examples are given that show that the limiting absolute sampling error, when measuring the radius of the round mark image, decreases with an increase in the number of pixels belonging to this image.

\section{RASTER GRAPHICS ANALYSIS OF A ROUND MARK IMAGE}

Figure 1 shows a raster graphics for the round mark image with a radius of 10 raster units.

The contour of a continuous round mark image is shown in dotted lines. This image lies on the grid formed by the elements of the raster matrix (pixels) of the recording device. As a result,

*Corresponding author's e-mail: a_grigorev@mail.ru 
a continuous image of the round mark is converted into its discrete image. It is the discrete image that is perceived by a measuring system. The discrete image differs from the continuous one in that the first can only include an integer number of pixels. Each particular element of a raster matrix cannot belong to a discrete image in part: either it belongs or does not belong to a discrete image entirely. If most of the pixel is within a continuous image, then the discrete image includes the entire pixel. If most of the pixel is outside the continuous image, then the entire pixel remains outside the discrete image [15]-[17].

The method for line number counting of a raster matrix intersecting a discrete mark image is that the number of such lines $n_{1}$ is initially counted, and then the radius of this image is calculated, measured by the method of counting lines $r_{\mathrm{cl}}$ by the following formulas:

$$
n_{1}=i_{\max }-i_{\min }+1
$$

where $i_{\max }$ and $i_{\min }$ are the largest and smallest values of the ordinal number of the raster matrix line, respectively, that intersects the mark image.

$$
r_{\mathrm{cl}}=\frac{n_{1}}{2}
$$

The value of $r_{\mathrm{cl}}$ is obtained in raster units (run).

The values for the round mark, shown in Fig. 1, are as follows:

$$
\begin{gathered}
i_{\text {max }}=26, i_{\text {min }}=6 \Rightarrow \\
n_{1}=i_{\text {max }}-i_{\text {min }}+1=26-6+1=21 \Rightarrow \\
r_{\mathrm{cl}}=\frac{n_{1}}{2}=\frac{21}{2}=10.5 \text { run. }
\end{gathered}
$$

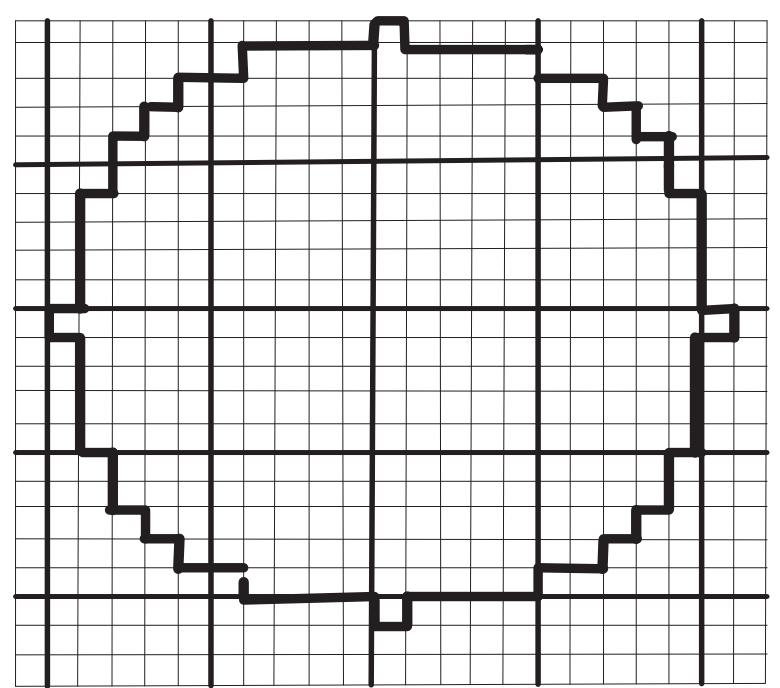

Fig. 1. Raster graphics for the round mark image.

The measurement error is the difference between the measured and reference value of the measured. The true value of the radius of the round mark image $r$ is the reference value:

$$
\varepsilon_{r \mathrm{cl}}=r_{\mathrm{cl}}-r,
$$

where $\varepsilon_{r \mathrm{cl}}$ is the radius measurement error of the round mark image by the method of line counting intersecting the image.

Thus, for the raster graphics in Fig. 1:

$$
\varepsilon_{r \mathrm{cl}}=r_{\mathrm{cl}}-r=10.5-10.0=0.5 \text { run. }
$$

The idea of the pixel counting method belonging to a discrete image of the round mark is as follows. First, the number of such $n_{\mathrm{p}}$ pixels is counted:

$$
n_{\mathrm{p}}=\sum_{i=1}^{I} \sum_{j=1}^{J} \xi(i ; j),
$$

where $i$ and $j$ are the sequence numbers of the line and column of the raster matrix, respectively; $I$ and $J$ are the number of lines and columns of the raster matrix, respectively; $\xi(i ; j)$ is the function that takes a value equal to one if the pixel of the $i$-th line of the $j$-th column of the raster matrix belongs to the image of the mark, and a zero, otherwise [18]-[20].

The area of each pixel $S_{\mathrm{p}}$ is equal to one square raster unit $\left(1 \mathrm{run}^{2}\right)$. It follows that $S_{\mathrm{cp}}=n_{\mathrm{p}} \cdot 1 \mathrm{run}^{2}$, where $S_{\mathrm{cp}}$ is the area of the round mark image, measured by the method of pixel counting. Thus, the area of the round mark image, measured by the pixel counting method in $\mathrm{run}^{2}$, is equal to the number of pixels belonging to this image.

After accumulation of the round mark image area, the value of its radius $r_{\mathrm{cp}}$ is computed by the method of pixel counting:

$$
r_{\mathrm{cp}}=\sqrt{\frac{S_{\mathrm{cp}}}{\pi}} .
$$

The radius measurement error of the round mark image by the pixel counting method is the follwing:

$$
\varepsilon_{r \mathrm{cp}}=r_{\mathrm{cp}}-r .
$$

The number of pixels of the round mark image, shown in Fig. 1, is the following:

$$
\begin{gathered}
n_{\mathrm{p}}=317 \Rightarrow S_{\mathrm{p}}=317 \mathrm{run}^{2} \Rightarrow \\
r_{\mathrm{cp}}=\sqrt{\frac{S_{\mathrm{cp}}}{\pi}}=\sqrt{\frac{317 \mathrm{run}^{2}}{\pi}}=10.045110 \text { run. }
\end{gathered}
$$

Hence, the radius measurement error $\varepsilon_{r \mathrm{cp}}$ of the round mark image by the pixel counting method is the following:

$$
\varepsilon_{r \mathrm{cp}}=r_{\mathrm{cp}}-r=10.045110-10=0.04511 \mathrm{run} \text {. }
$$

\section{MODELLING OF THE RADIUS MEASUREMENT ERROR OF THE ROUND MARK IMAGE}

As a result of modelling, the methods for measuring the radius of the round mark image with $r$ variation in the range from 9.5 run to 10.5 run, the graphs of the dependence of the radius measurement error of the round mark image on its true value are obtained (Fig. 2). 


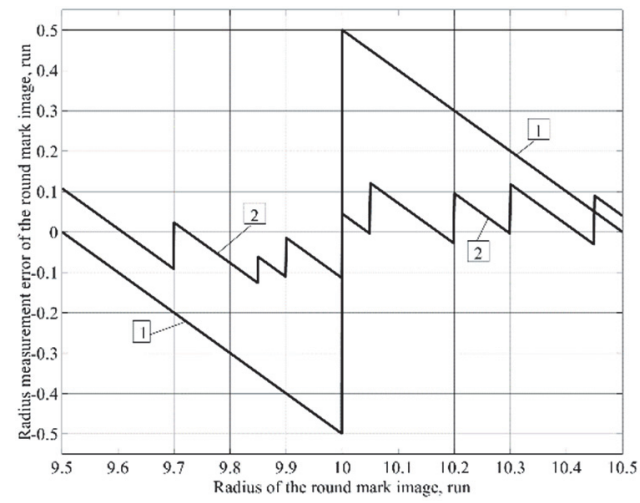

Fig. 2. The radius measurement error of the round mark image.

In Fig. 2, a broken line 1 corresponds to the radius measurement error $\varepsilon_{r \mathrm{cl}}$ of the round mark image by the line counting method, and a broken line 2 corresponds to the radius measurement error $\varepsilon_{r \mathrm{cp}}$ of the round mark image by the pixel counting method. The radius measurement error of the round mark image by the method of line counting varies between limiting values +0.5 run and -0.5 run. The radius measurement error of the round mark image by the method of pixel counting varies between the limiting values +0.1211 run and 0.1259 run.

The graph represented by the broken line 1 in Fig. 2 shows that the radius measurement error of the round mark image by the method of line counting is distributed between two limiting values: -0.5 run and +0.5 run. Thus, the limiting absolute error of measuring the radius of the round mark image is 0.5 run. The limiting absolute error of measuring the radius of the round mark image by the method of line counting does not depend on the value of the radius of the round mark image. If, for example, the true radius of the round mark image is not ten, but one hundred raster units, the measurement error will be equally distributed between the same two limiting values +0.5 run and -0.5 run.

As for the pixel counting method, the limiting absolute error in radius measuring of the round mark image depends on the value of this radius: the larger the radius of the image of the mark is, the smaller the absolute error of its measurement is.

\section{Method for Calculating the Limiting AbSOlute ERROR IN MEASURING THE RADIUS OF THE ROUND MARK IMAGE, AND GAINING FROM THE APPLICATION OF PIXEL COUNTING METHOD}

In [2], [3] it is shown that

$$
\Delta S_{\mathrm{cp}}=k_{\Delta S \mathrm{cp}} \sqrt{r_{\mathrm{cp}}},
$$

where $\Delta S_{\mathrm{cp}}$ is the limiting absolute error of area measuring of the round mark image by the pixel counting method with a confidence probability of 0.9973 ; the coefficient $k_{\Delta S \mathrm{sp}}$ is a constant:

$$
k_{\Delta S \mathrm{cp}}=\sqrt[4]{4.5 \pi^{2}} \operatorname{run}^{3 / 2} .
$$

A coverage interval is the interval based on available information, which contains a set of true values of the measured quantity with a given probability.

Thus, the boundaries of the area coverage interval of the round mark image with a confidence probability of 0.9973 are:

$$
\begin{aligned}
& S_{\text {cp min }}=S_{\text {cp }}-\Delta S_{\mathrm{cp}}, \\
& S_{\text {cp max }}=S_{\text {cp }}+\Delta S_{\text {cp }},
\end{aligned}
$$

where $S_{\mathrm{cp} \max }$ and $S_{\mathrm{cp} \text { min }}$ are the upper and lower boundaries of the area coverage interval of the round mark image, respectively.

It follows from (7), (13) and (14) that:

$$
\begin{gathered}
r_{\text {cp max }}=\sqrt{\frac{S_{\text {cp max }}}{\pi}}, \\
r_{\text {cp min }}=\sqrt{\frac{S_{\mathrm{cp} \min }}{\pi}},
\end{gathered}
$$

where $r_{\mathrm{cp} \text { max }}$ and $r_{\mathrm{cp} \text { min }}$ are the upper and lower boundaries of the radius coverage interval of the round mark image, respectively.

In order to estimate the limiting absolute error of radius measuring of the round mark image $\Delta r_{\mathrm{cp}}$, calculations should be performed using (17)-(19):

$$
\Delta_{\mathrm{U}} r_{\mathrm{cp}}=r_{\mathrm{cp} \max }-r_{\mathrm{cp}},
$$

where $\Delta_{\mathrm{U}} r_{\mathrm{cp}}$ is the difference between the upper interval boundary of the radius coverage of the round mark image, and its measured value.

$$
\Delta_{\mathrm{L}} r_{\mathrm{cp}}=r_{\mathrm{cp}}-r_{\mathrm{cp} \min },
$$

where $\Delta_{\mathrm{L}} r_{\mathrm{cp}}$ is the difference between the measured radius value of the round mark image, and the lower boundary of its coverage interval.

$$
\Delta r_{\text {cp }}=\left\{\begin{array}{l}
\Delta_{\mathrm{U}} r_{\mathrm{cp}}, \text { if } \Delta_{\mathrm{U}} r_{\mathrm{cp}} \geq \Delta_{\mathrm{L}} r_{\mathrm{cp}} \\
\Delta_{\mathrm{L}} r_{\mathrm{cp}}, \text { if } \Delta_{\mathrm{U}} r_{\mathrm{cp}}<\Delta_{\mathrm{L}} r_{\mathrm{cp}} .
\end{array}\right.
$$

In the example considered in the present article, the number of pixels belonging to the round mark image is 317 . The measured value of the round mark image radius calculated by (7) is: $r_{\mathrm{cp}}=10.045110$ run. Calculations according to (7)-(14) showed that the limiting absolute error of radius measuring of the round mark image by the pixel counting method with a confidence probability of 0.9973 is: $\Delta r_{\mathrm{cp}}=0.1305$ run. The absolute values of all measurement errors obtained by modelling do not exceed this value of the limiting absolute error. Evaluation of the gain accuracy in radius measuring of the round mark image by the limiting absolute errors using the pixel counting method, in relation to the use of the line counting method $p_{\Delta r \text { cpl }}$ is determined by the formula:

$$
p_{\Delta r \mathrm{cpl}}=\frac{\Delta r_{\mathrm{cl}}}{\Delta r_{\mathrm{cp}}}
$$


V. ANALYSIS OF SPECIFIC CALCULATION EXAMPLES OF THE LiMITING ABSOLUTE ERROR IN RADIUS MEASURING OF THE Round MARK IMAGE, AND THE GAIN FROM APPLYING THE Pixel COUNTING METHOD

The limiting absolute error in radius measuring of the round mark image by the line counting method, regardless of the number of pixels belonging to the round mark image, is $\Delta r_{\mathrm{cl}}=0.5$ run. If the number of pixels belonging to the round mark image is 317 , then the gain in the radius measuring accuracy of the round mark image using the pixel counting method, calculated by (15), is:

$$
p_{\Delta r \mathrm{cpl}}=\frac{\Delta r_{\mathrm{cl}}}{\Delta r_{\mathrm{cp}}}=\frac{0.5 \text { run }}{0.1305 \text { run }}=3.8319 .
$$

Such a limiting absolute error and such a gain in accuracy is provided by the pixel counting method, with a number of pixels belonging to the round mark image of $n_{\mathrm{p}}=317$.

This $n_{\mathrm{p}}$ value is obtained from the modelling. The actual number of pixels belonging to the round mark image is much larger. Thus, for example, for a real monitoring of the distribution of vibrational displacements of material points of the test object surface, a matrix of round marks is placed on this surface, in the geometric centres of which the material points under study are located (Fig. 3).

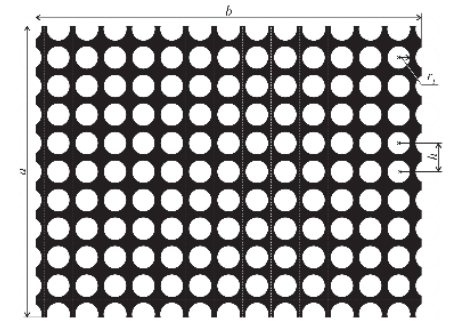

Fig. 3. A fragment of the matrix of round marks in the field of view of the recording device: $h$ is the distance between the centres of round marks in the lines and columns of the matrix; $r_{\mathrm{t}}$ is the radius of the round mark; $a$ and $\mathrm{b}$ are the smaller and larger sides of the rectangular field of view of the recording device, respectively; $R_{\mathrm{CAM}}$ is the resolution of the recording device.

The image formed by the recording device is similar to the displayed original one. It follows that

$$
\frac{S_{\mathrm{f}}}{S_{\mathrm{t}}}=\frac{R_{\mathrm{CAM}}}{n_{\mathrm{p}}},
$$

where $S_{f}$ is the field of view area of the recording device; $S_{t}$ is the area of the round mark.

Consequently:

$$
n_{\mathrm{p}}=\frac{S_{\mathrm{t}} R_{\mathrm{CAM}}}{S_{\mathrm{f}}} .
$$

According to Fig. 3:

$$
S_{\mathrm{f}}=a b ; S_{\mathrm{t}}=\pi r_{\mathrm{t}}^{2}
$$

From here:

$$
n_{\mathrm{p}}=\frac{\pi r_{\mathrm{t}}^{2} R_{\mathrm{CAM}}}{a b} \text {. }
$$

If, for example, $h=5 \mathrm{~mm} ; r_{\mathrm{t}}=2 \mathrm{~mm} ; a=75 \mathrm{~mm}$; $b=100 \mathrm{~mm} ; R_{\mathrm{CAM}}=5$ Mpix, then:

$$
n_{\mathrm{p}}=\frac{\pi r_{\mathrm{t}}^{2} R_{\mathrm{CAM}}}{a b}=\frac{\pi \cdot 2^{2} \cdot 5 \cdot 10^{6}}{75 \cdot 100}=8378 .
$$

It follows that the area value of the mark image, measured by the pixel counting method, is $\mathrm{S}_{\mathrm{cp}}=8378 \mathrm{run}^{2}$. The radius value of the mark image, calculated by (5), is $r_{\mathrm{cp}}=51.64$ run. The value of the limiting absolute error of radius measuring of the round mark image, calculated by (11)-(19) using the pixel counting method with a confidence probability of 0.9973 , is: $\Delta r_{\mathrm{cp}}=0.05721$ run. The gain in accuracy in comparison with the line counting method, calculated according to (15), is:

$$
p_{\Delta r \mathrm{cpl}}=\frac{\Delta r_{\mathrm{cl}}}{\Delta r_{\mathrm{cp}}}=\frac{0.5 \text { run }}{0.05721 \mathrm{run}}=8.7403 .
$$

If, for example, it is required to measure the vibrational displacement of one of the studied points with a higher accuracy, then the recording device can be focused on one of the matrix marks (Fig. 4).

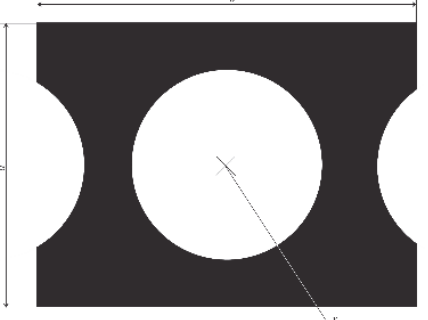

Fig. 4. One mark in the field of view of the recording device.

In this case, all parameters of the matrix of round marks remain unchanged, except for the parameters of the field of view of the recording device: $a=6 \mathrm{~mm} ; b=8 \mathrm{~mm}$. Calculations with these parameters using (27) show that:

$$
n_{\mathrm{p}}=\frac{\pi r_{\mathrm{t}}^{2} R_{\mathrm{CAM}}}{a b}=\frac{\pi \cdot 2^{2} \cdot 5 \cdot 10^{6}}{6 \cdot 8}=1308997
$$

It follows from this consideration that the mark image area is $S=1308997$ run $^{2}$. The radius value of the mark image calculated from (7) is $r_{\mathrm{cp}}=645.5$ run. The value of the limiting absolute error in measuring the radius of the round mark image calculated from (11)-(19) by the method of counting pixels with a confidence probability of 0.9973 will be: $\Delta r_{\mathrm{cp}}=0.01617$ run. The gain in accuracy in comparison with the method of counting lines, calculated by (15), will be:

$$
p_{\Delta r \mathrm{cpl}}=\frac{\Delta r_{\mathrm{cl}}}{\Delta r_{\mathrm{cp}}}=\frac{0.5 \text { run }}{0.01617 \text { run }}=30.92 \text {. }
$$




\section{CONCLUSION}

Modelling of the error in measuring the radius of the round mark image by the method of counting the number of raster lines, intersecting this image, and by the method of counting the number of pixels, belonging to this image, has been conducted in the present study. The basis of the modelling technique is the construction and analysis of the image raster graphics. A specific numerical example has also been considered in the paper.

The graphs of the dependence of the error in measuring the radius of the round mark image on the value of this radius have been constructed. Estimations of the limits of the error coverage intervals of measurement results have been carried out.

The limiting absolute error in measuring the radius of the round mark image has been estimated by both compared methods, depending on the number of pixels per image of the round mark. It is proved that the gain in the accuracy of measuring the radius of the round mark image when applying the method of counting the number of pixels belonging to this image can be more than thirtyfold. The formulas proposed in the article are used for software implementation of non-contact vibration measurement systems.

\section{ACKNOWLEDGMENT}

The results published in the article have been obtained working for State Contract No. 5917p/8265 "Research of Characteristics of Beam Splitters" with the Russian Federal State Budgetary Institution "Foundation for Assistance to Small Innovative Enterprises in the Scientific and Technical Sphere".

\section{REFERENCES}

[1] G. V. Tankov, S. A. Brostislov, N. K. Yurkov, and A. V. Lysenko, "Information-Measuring and Operating Systems to Test for the Effects of Vibration," in 2016 International Siberian Conference on Control and Communications (SIBCON), 2016. https://doi.org/10.1109/sibcon.2016.7491679

[2] A. V. Grigorev, V. A. Trusov, V. Ya. Bannov, P. G. Andreev, and G. V. Tan'kov, "Simulation of the Blurred Track of the Round Mark Image in Its Coplanar and Orthogonal Vibrational Displacements," in Proc. International Symposium Reliability and Quality, 2015, vol.1, pp. 107-109.

[3] N. K. Yurkov, G. V. Tankov, A. V. Lysenko, and V. A. Trusov, "On the Problem of Experimental Research of Forced Vibrations of Plates," in Proc. 19th International Conference on Soft Computing and Measurements (SCM), 2016, pp. 416-418. https://doi.org/10.1109/scm.2016.7519798

[4] A. V. Grigorev, A. K. Grishko, N. V. Goryachev, N. K. Yurkov, and A. M. Micheev, "Contactless Three-Component Measurement of Mirror Antenna Vibrations," in Proc. 2016 International Siberian Conference on Control and Communications (SIBCON), 2016. https://doi.org/10.1109/sibcon.2016.7491673

[5] A. V. Grigoriev, E. A. Danilova, and N. K. Yurkov, "Method of Measuring Vibration Movements of Material Points on the Surface of the Controlled Object," in Proc. 2017 20th IEEE International Conference on Soft Computing and Measurements (SCM), 2017. https://doi.org/10.1109/scm.2017.7970659

[6] A. V. Grigoriev, N. K. Yurkov, and I. I. Kochegarov, "Contactless Measurement Technique for the Amplitude of Vibrational Movement of the Test Material Point," in Proc. IEEE East-West Design \& Test Symposium (EWDTS'2016), pp. 549-551. https://doi.org/10.1109/ewdts.2016.7807737
[7] V. R. Roganov, E. A. Asmolova, A. N. Seredkin, M. V. Chetvergova, N. B. Andreeva, and V. O. Filippenko, "Problem of virtual space modelling in aviation simulators," Life Science Journal, vol. 11. no. 12s, pp. 371-373, 2014. Available: http://www.penzgtu.ru/fileadmin/ filemounts/its/staff/publish/filippenko/p3.pdf

[8] A. V. Dubravin, S. A. Zinkin, and D. V. Paschenko, "Formal and conceptual definitions of the hybrid model of distributed computings in networks," in 2015 International Siberian Conference on Control and Communications (SIBCON), May 2015. https://doi.org/10.1109/sibcon.2015.7147047

[9] M. Y. Mikheev, V. R. Roganov, P. G. Andreev, N. V. Goryachev, and V. A. Trusov, "Developing the Structure of the Quality Control System of Power Supply Units in Mobile Robots," in Proc. 2017 International Siberian Conference on Control and Communications (SIBCON), 2017. https://doi.org/10.1109/sibcon.2017.7998579

[10] V. R. Roganov, A. B. Sagyndyk, R. F. Akhtarieva, A. K. Beisenbayeva, and S. I. Sannikova, "Integrated Organization of the System for Forming the Information Support of Aeronautical Simulator," in International Journal of Applied Engineering Research, vol. 12, no. 15, pp. 52075213,2017

[11] J. Lénárt, "Contactless Vibration Measurement Using Linear CCD Sensor," in Proc. 13th International Carpathian Control Conference (ICCC), High Tatras, 2012, pp. 426-429. https://doi.org/10.1109/carpathiancc.2012.6228681

[12] H. J. Tiziani, "Optical Methods for Precision Measurements," Optical and Quantum Electronics, vol. 21, pp. 253-282, 1989.

[13] K. R. McCall, M. Boudjema, I. B. Santos, R. A. Guyer, and G. N. Boitnott, "Nonlinear, Hysteretic Rock Elasticity: Deriving Modulus Surfaces," in The 38th U.S. Symposium on Rock Mechanics (USRMS), Washington, July 7-10, 2001.

[14] V. Roganov, M. Miheev, E. Roganova, B. I. Nurgozhin, and V. Fillipenko, "Main Provisions for Formation of Cognitive Model of Visually Observable Environment Synthesized for Aircraft Simulator" in Proc. International Conference "Actual Issues of Mechanical Engineering” (AIME), 2017, pp. 671-676.

[15] C. Ding, J. L. G. Janssen, A. A. H. Damen, P. P. J. van den Bosch, J. J. H. Pau-lides, and E. Lomonova, "Modeling and Realization of a 6-DoF Contactless Electromagnetic Anti-Vibration System and Verification of Its Static Behavior," in IEEE/ASME International Conference on Advanced Intelligent Mechatronics (AIM), Kachsiung, 2012, pp. 149154. https://doi.org/10.1109/aim.2012.6265880

[16] A. Grishko, I. Kochegarov, and N. Yurkov, "Structural and Parameter Optimization of the System of Interconnected Processes of Building Complex Radio-Electronic Devices," in Proc. 14th International conference CADSM, Lviv, Ukraine, 2017, pp. 192-194. https://doi.org/10.1109/cadsm.2017.7916112

[17] A. K. Grishko, N. V. Goryachev, I. I. Kochegarov, and M. P. Kalaev, "Mathematical Models of the System of Measurement and Analysis of Temperature Parameters of Radio Electronic Modules," in 2016 13th International Scientific-Technical Conference on Actual Problems of Electronics Instrument Engineering (APEIE), Novosibirsk, Russia, October 3-6, 2016, vol. 2, pp. 112-115, https://doi.org/10.1109/apeie.2016.7806424

[18] E. Y. Maksimov, N. K. Yurkov, and A. N. Yakimov, "A Finite-Element Model of the Thermal Influences on a Microstrip Antenna," Measurement Techniques, vol. 54, no. 2, pp. 207-212, May 2011. https://doi.org/10.1007/s11018-011-9707-y

[19] E. V. Lapshin, N. K. Yurkov, and S. A. Brostilov, "Implementation of Systems Approach to Identification and Quality Management," in Proc. 14th International Conference CADSM, Lviv, Ukraine, 2017, pp. 188 191. https://doi.org/10.1109/cadsm.2017.7916111

[20] G. V. Tankov, S. A. Brostislov, N. K. Yurkov, and A. V. Lysenko, "Information-Measuring and Operating Systems to Test for the Effects of Vibration," in 2016 International Siberian Conference on Control and Communications (SIBCON), 2016. https://doi.org/10.1109/sibcon.2016.7491679 


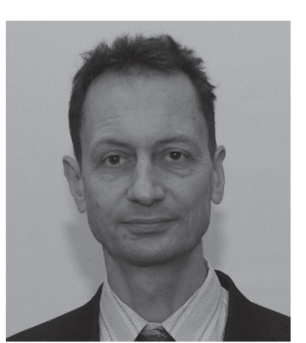

Alexey Grigoriev graduated from Penza Polytechnic Institute in the specialty "Design and Manufacture of Radio Equipment" in 1981. Research interests: processing and automated analysis of raster images. In 1999, he defended his thesis for the direct registration and processing of electron diffraction patterns. $\mathrm{He}$ is a Candidate of Technical Sciences. E-mail: a grigorev@mail.ru

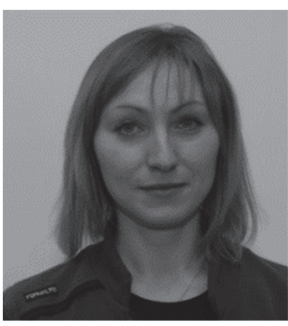

Evgeniya Danilova graduated from Penza State University in 2002 (Penza, Russia), receiving qualification in "Design and Technology of Radio-Electronic Equipment". At present, she is an Associate Professor at the Department of Development and Production of Radio-Electronic Equipment at Penza State University. She defended her Master's Thesis in 2015.

She is an active participant of the research projects at the department. The object of the research is increasing the reliability of radio-electronic equipment and quality assessment in PCB production. She is the author of more than 30 research papers and conference reports in various journals.

She is currently conducting her post-graduate research.

E-mail: siori@list.ru

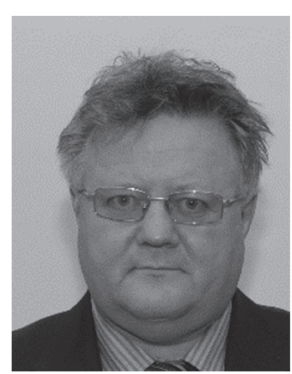

Vasiliy Trusov graduated from Penza Polytechnical Institute in the speciality "Designing and Production of Radio Equipment" in 1982. Since August 1994, he has engaged in teaching and scientific research in Penza State University. Research interests: technology, computer science. In 1997 he defended his thesis. The courses taught: "Computer Graphics"; "Theory of Oscillations"; "Electronics"; "Optimisation Techniques"; "Micro- and Nanotechnology Production of RES". He is a Candidate of Technical Sciences, Associate Professor. He has over 100 scientific papers, 5 patents.

E-mail: trusov_v@mail.ru

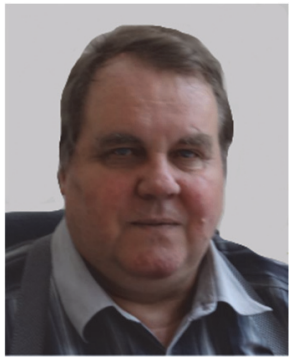

Mikhail Mikheev graduated from Penza Polytechnic Institute (1981) (postgraduate and doctoral studies at Penza Polytechnic Institute), defended his thesis for the degree of Candidate of Technical Sciences (1986) and Doctor of Technical Sciences (2002). He is the author of more than 200 scientific papers.

$\mathrm{He}$ is a Professor at the Department of Information Technologies and Systems of Penza State Technological University. He is a Leading Specialist of Video3 LLC (since 2009).

He is an Academician of the International Academy of Informatization of Education.

Address: Volodarsky Str. 74, ap. 2, Penza, 440000, Russia.Phone: +7 906 3959587

E-mail: mix1959@gmail.com

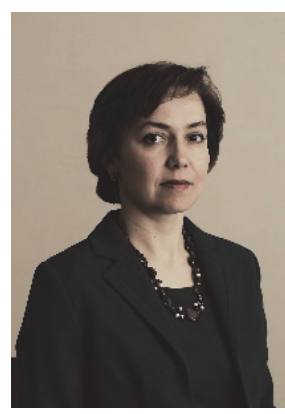

Marina Uhanova graduated from Riga Aviation University, receiving the Bachelor's Degree in 1995 and Master's Degree in 1996. In 2007, she received the Doctoral Degree in System Analysis, Modelling and Development from Riga Technical University. Since 2000, she has worked as an Assistant and Lecturer. Her current employment is an Assistant Professor at the Chair of Software Engineering, Riga Technical University. Her research interests include distributed application development and their applications in insurance.

E-mail: marina.uhanova@rtu.lv 